\title{
Maximum Lifetime Paths for the High Packet Delivery Ratio Using Fast Recovery in a Mobile Ad Hoc Network*
}

\author{
HyoJin Kim, SeungJae Han, and JooSeok Song \\ Department of Computer Science, Yonsei University, Seoul 120-749, Korea \\ hyojin@emerald.yonsei.ac.kr, sjhan@cs.yonsei.ac.kr, \\ jssong@emerald.yonsei.ac.kr
}

\begin{abstract}
Both movement of mobile nodes and path lifetime exhaustion cause to change the network topology in a mobile ad hoc network. Then, it is not easy to obtain the high packet delivery ratio. This paper proposes the Maximum Lifetime Paths (MLP) to transmit a large amount of data using nodes with long lifetime and using fast recovery. The proposed MLP establishes routes based on the shortest path and dynamically recovers routes using warning messages. The simulation experiment results show that MLP increases the packet delivery ratio and reduces the hop distance and the frequency of the route establishment.
\end{abstract}

\section{Introduction}

In a mobile ad hoc network, mobile nodes communicate with each others having the limited battery power without any infrastructure. Thus, both node movement and path lifetime exhaustion cause to change the network topology. Dynamic Source Routing (DSR) is one of routing protocols in a mobile ad hoc network. Although DSR can provide the packet salvaging and multiple routes, it usually uses only one route to transmit packets which we call to DSR1 [1]. However, the traditional routing protocols including DSR1 take only node movement into consideration. Thus, recently many researchers have studied about the path lifetime extension, for example, the Power-Aware Routing Technique (PARO) [2] and the Conditional Max-Min Battery Capacity Routing (CMMBCR) [3]. However, PARO and CMMBCR do not take fast recovery into consideration. Therefore, this paper proposes the Maximum Lifetime Paths (MLP) to transmit a large amount of data by nodes with high energies and fast recovery. The proposed MLP based on DSR establishes routes based on the minimum hops (i.e. the shortest path) and dynamically recovers routes using Route Warning (RWAR) message.

The rest of paper is as follows: Section 2 describes MLP, and Section 3 presents the performance analysis. Finally, Section 4 concludes the paper.

* This research was supported by the MIC(Ministry of Information and Communication), Korea, under the ITRC(Information Technology Research Center) support program supervised by the IITA(Institute of Information Technology Assessment). 


\section{Maximum Lifetime Paths (MLP)}

Like DSR, MLP finds the shortest path from source to destination using Route Request (RREQ)-Route Reply (RREP) cycles. We choose nodes that have longer lifetime than the Reference Point $(R P)$ to extend the path lifetime as the following steps:

Step 1. The source $(S)$ calculates the appropriate number of nodes with the normalized node lifetime and finds the average value $(E)$. $S$ chooses $R P$ as $E-\frac{M A X-E}{2}$ when $M A X$ is the longest normalized node lifetime. Each node $\left(n_{i}\right)$ including $S$ compares the lifetime of the next node $\left(n_{i+1}\right)$ with $R P$.

Step 2.1 If the lifetime of $n_{i+1}$ is longer than $R P, n_{i}$ uses the link to $n_{i+1}$.

Step 2.2 Otherwise, $n_{i}$ compares the lifetime of the following nodes such as $n_{i+2}$, $n_{i+3}$, and so forth on the primary route with $R P$ and chooses a new alternative route to the chosen node.

Step 2.2.1 If the alternative route is existed and the path lifetime is longer than $R P$, $n_{i}$ uses the route to forward packets. The path lifetime of the alternative route is determined by the node that has the minimum lifetime on the path. If the path lifetime of the alternative route is not longer than $R P$, $n_{i}$ chooses another route to the chosen node.

Step 2.2.2 Otherwise, go to Step 2.1.

Step 3. $S$ forwards packets using the established route. $n_{i}$ establishes another disjoint alternative route to $n_{i+2}$ or the following node and reserves the route if $n_{i}$ can.

When the lifetime of $n_{i+1}$ is shorter than $R P, n_{i+1}$ sends a RWAR to $n_{i} n_{i}$ compares the path lifetime of the reserved route with $R P$. If the lifetime of the reserved route is longer than $R P, n_{i}$ uses the reserved route. If there is no reserved route in $n_{i}$ or the path lifetime of the reserved route is not longer than $R P, n_{i}$ chooses another route to $n_{i+2}$ or the following nodes such as $n_{i+3}, n_{i+4}$, and so on. Using the new alternative route, $n_{i}$ forwards the later packets. In this way, MLP protects the path lifetime exhaustion.

When $n_{i+1}$ moves out of the network during data transmissions, route maintenance is started by sending a Route Error (RERR) to $n_{i}$. $n_{i}$ forwards the later packets to the reserved alternative route immediately, establishes another disjoint route with the reserved route, and reserves the route. If there is no reserved route from $n_{i}, n_{i}$ forwards the RERR to $S$. In this way, MLP maintains routes against the node movement.

\section{Performance Analysis}

The packet delivery ratio $(P D R)$ is defined as the ratio between the amount of packets sent by the source and received by the destination. We assume that packets are transmitted during time from $t_{s}$ to $t_{d}$ with the generating rate $(p)$ and the receiving rate $(q)$. When we assume that the number of nodes on the primary route in MLP is $l$, the path lifetime of the primary route $\left(f_{1}\right)$ of MLP is as the following:

$$
f_{1}=\min \left\{i_{0}, i_{1}, i_{2}, i_{3}, \cdots, i_{l}\right\} .
$$


When we denote $f_{0}$ to a failure time due to the node movement, a failure occurs in MLP as:

$$
f_{M L P}=\min \left\{f_{0}, f_{1}\right\} .
$$

Therefore, $P D R$ of MLP $\left(P D R_{M L P}\right)$ satisfies the following equation:

$$
P D R_{M L P}=\frac{\int_{t_{s}}^{t_{d}}(q) d t}{\int_{t_{s}}^{t_{d}}(p) d t}=\frac{\int_{t_{s}}^{f_{M L P}}(q) d t+\int_{f_{M L P}}^{t_{d}}(q) d t}{\int_{t_{s}}^{t_{d}}(p) d t} .
$$

We simulate 50 mobile nodes in a $1000 \mathrm{~m}$ by $1000 \mathrm{~m}$ area with 10 sources for 900 seconds focusing on PDR using Lucent $2 \mathrm{Mb} / \mathrm{s}$ WaveLAN 802.11 Lancard by ns-2 simulations [4]. Each node uses the random waypoint model and moves with a speed from 0 to $5 \mathrm{~m} / \mathrm{s}$. We change the pause time when every node is initially stopped. The Constant Bit Rate (CBR) sources send 512-byte User Datagram Protocol (UDP) packets with 5 packets/sec. The lifetime of each node is normalized from 0 to 1.
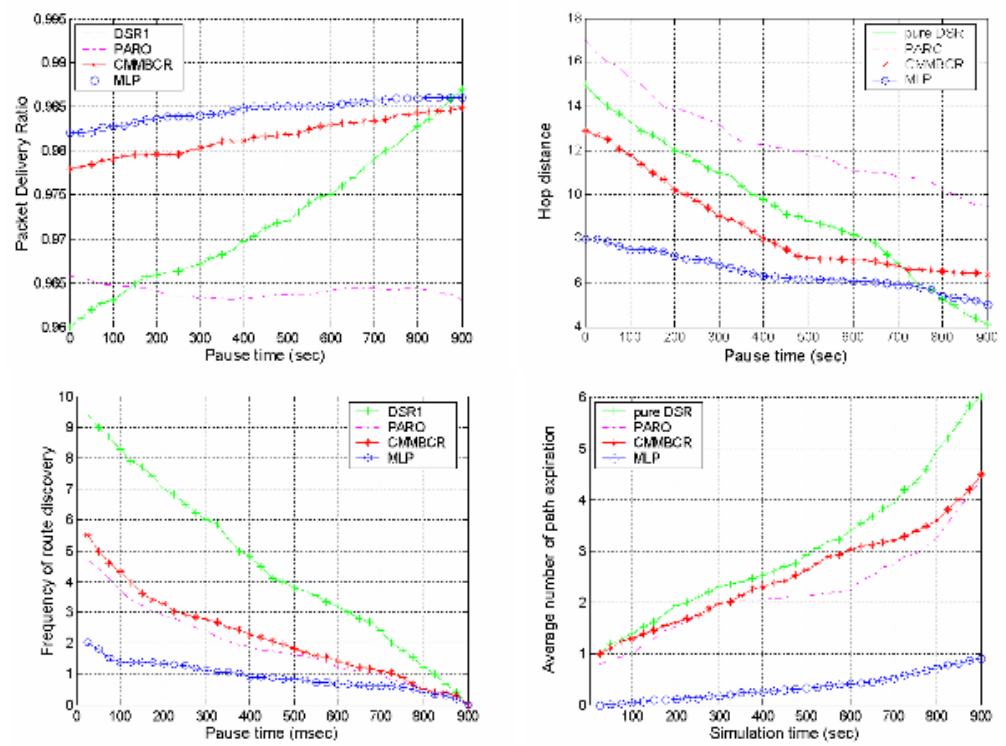

Fig. 1. (a) Packet delivery ratio vs. pause time (b) Hop distance vs. pause time (c) Frequency of route discovery vs. pause time (d) Average number of path expiration vs. simulation time

Fig. 1(a) shows that MLP has higher PDR than other protocols in high mobility. When mobile nodes are stopped, DSR1 has the highest PDR using the shortest path and PARO has the lowest $P D R$ having additional hops for redirectors. The hop distance is defined as the number of hops that packets traverse using the shortest path before detecting a failure and the alternative route after detecting a failure. Fig. 1(b) presents that MLP has the shortest hop distance in high mobility. CMMBCR extends node lifetime but uses longer hops than MLP in high mobility because it does not 
provide fast recovery. In Fig. 1(c), MLP initiates the fewest route discoveries in high mobility, but DSR1 discovers routes frequently in high mobility because it does not reserve any alternative route. Both CMMBCR and PARO have to use route discovery in high mobility because they do not dynamically recover routes. Fig. 1(d) describes the average number of path expiration with varying simulation time. The path expiration is defined as the lifetime exhaustion of the path from source to destination. The paths of MLP are nearly expired contrary to DSR1.

\section{Conclusion}

This paper has proposed a new routing protocol for the high packet delivery ratio against both node movement and path lifetime exhaustion even though the proposed protocol has control overheads to establish local alternative routes dynamically. The simulation results show that the proposed protocol is more efficient than the existing protocols such as $1.26 \%, 2.08 \%$, and $0.30 \%$ than DSR1, PARO, and CMMBCR.

\section{References}

1. Lee, S.-J., et. al.: Split multipath routing with maximally disjoint paths in ad hoc networks. IEEE ICC 2001, Vol. 10. (2001) 3201-3205

2. Comez, J., et. al.: PARO: Supporting Dynamic Power Controlled Routing in Wireless Ad Hoc Networks. Wireless Networks. Kluwer Academic Publishers, Netherlands (2003) 443-460

3. Toh, C.-K.: Maximum Battery Life Routing to Support Ubiquitous Mobile Computing in Wireless Ad Hoc Networks. IEEE Communications Magazine (2001) 2-11

4. Chen, B., et. al.: Span: An Energy-Efficient Coordination Algorithm for Topology Maintenance in Ad Hoc Wireless Networks. Wireless Networks, Vol. 8 (2002) 481-494 